\title{
Task set induces dynamic reallocation of resources in visual short-term memory
}

\author{
Summer L. Sheremata ${ }^{1,2} \cdot$ Sarah Shomstein ${ }^{3}$
}

Published online: 3 January 2017

(C) Psychonomic Society, Inc. 2016

\begin{abstract}
Successful interaction with the environment requires the ability to flexibly allocate resources to different locations in the visual field. Recent evidence suggests that visual short-term memory (VSTM) resources are distributed asymmetrically across the visual field based upon task demands. Here, we propose that context, rather than the stimulus itself, determines asymmetrical distribution of VSTM resources. To test whether context modulates the reallocation of resources to the right visual field, task set, defined by memory-load, was manipulated to influence visual short-term memory performance. Performance was measured for single-feature objects embedded within predominantly single- or two-feature memory blocks. Therefore, context was varied to determine whether task set directly predicts changes in visual field biases. In accord with the dynamic reallocation of resources hypothesis, task set, rather than aspects of the physical stimulus, drove improvements in performance in the right- visual field. Our results show, for the first time, that preparation for upcoming memory demands directly determines how resources are allocated across the visual field.
\end{abstract}

Keywords Visual working memory · Cognitive and attentional control $\cdot$ Short term memory

Summer L. Sheremata

ssheremata@fau.edu

1 Department of Psychology, Florida Atlantic University, 777 Glades Road, Boca Raton, FL 33487, USA

2 Center for Complex Systems and Brain Sciences, Florida Atlantic University, Boca Raton, FL 33487, USA

3 Department of Psychology, George Washington University, Washington, DC 20052, USA

\section{Introduction}

Interacting with a dynamically changing world requires the ability to continuously represent visual information across time and distraction. Visual short-term memory (VSTM) is a memory system that maintains visual information for brief durations, allowing for the continuous representation of objects in the visual world (Phillips, 1974). However, VSTM is severely limited in capacity, estimated at approximately $3 \pm 1$ simple objects (Alvarez \& Cavanagh, 2004; Luck \& Vogel, 1997).

The severe capacity limits in VSTM reflect constraints in the processing capabilities of underlying brain networks (Cowan, 2010; Phillips, 1974), mainly those involving the intraparietal sulcus (IPS). Evidence from human neuroimaging and electrophysiological studies have converged on a central role of IPS in the encoding and maintenance of information in VSTM (Todd \& Marois, 2004; Vogel \& Machizawa, 2004; Xu \& Chun, 2006). In contrast to the visual system, which represents items strictly in the contralateral visual field, IPS represents spatial locations in a hemisphere- and taskdependent manner (Sheremata \& Silver, 2015; Sheremata, Bettencourt, \& Somers, 2010; Szczepanski, Konen, \& Kastner, 2010), allowing cognitive resources to be allocated based upon current task demands. Specifically, increasing the number of items held in VSTM results in whole field representations by right, but not left, IPS (Sheremata et al., 2010).

Consistent with a relationship between brain activity and behavioral performance, visual field asymmetries have been linked to asymmetries in the brain. For example, it has been demonstrated that hemispheric asymmetries seen in VSTM result in behavioral asymmetries across the visual field that are dependent on the number of features to be remembered (Sheremata \& Shomstein, 2014). Namely, it was demonstrated that there is an advantage for items held in VSTM in the left 
visual field when encoding and storing a single feature. These results are consistent with a right-hemisphere bias observed in the neuroimaging literature, resulting in better performance for items in the left visual field during both visual attention (Bowers \& Heilman, 1980; Thiebaut de Schotten et al., 2011) and working memory (Gamble \& Somers, 2012).

Memory demand is determined not only by the number of items remembered, but also by the number of visual features in each remembered item (Alvarez \& Cavanagh, 2004; Fougnie, Asplund, \& Marois, 2010; Wilson, Adamo, Barense, \& Ferber, 2012; Xu \& Chun, 2006), but see (Luck $\&$ Vogel, 1997). Consistent with these studies, VSTM performance was impaired when participants were required to maintain two features in memory. Furthermore, in this condition visual field asymmetries reverse, demonstrating a right visual field benefit (Sheremata \& Shomstein, 2014). This reversal is again predicted by brain imaging in healthy individuals that demonstrate whole field representations emerge with increased attention (Sheremata \& Silver, 2015; Szczepanski et al., 2010) and memory demands (Sheremata et al., 2010).

To explain behavioral asymmetries in resource allocation between the left and right visual fields, we proposed the dynamic reallocation hypothesis. This hypothesis borrows heavily from the notion of right hemisphere dominance in visuo-spatial processing (Mesulam, 1999), and is consistent with decades of investigations on attentional asymmetries observed in visuo-spatial neglect (Behrmann, Watt, Black, \& Barton, 1997; Bisiach \& Vallar, 1988; Eglin, Robertson, \& Knight, 1989; Kinsbourne, 1987; Posner, Walker, Friedrich, \& Rafal, 1984). According to the dynamic reallocation hypothesis, the left hemisphere has limited resources to allocate to the right visual field. Therefore, when task demands require greater resources in the right visual field than are available from the left hemisphere, the right hemisphere dynamically reallocates resources to the right visual field. This is supported by findings that increasing attentional (Poynter \& Roberts, 2012) and memory (Sheremata \& Shomstein, 2014) demands results in a benefit for right visual field representations.

It is possible that hemispheric asymmetries may modulate performance across the visual field even when differences in behavior across the visual field are not apparent. VSTM performance reflects various cognitive demands, such as attending to the memory items, ignoring distracting information, and comparing remembered objects to the memory probe. It is likely that only some of these cognitive functions are lateralized in the brain, and that VSTM performance at various memory set sizes reflects increased demands on lateralized and non-lateralized functions. Therefore, modulating memory demand via set size may obscure visual field asymmetries that affect memory performance differentially in the left- and rightvisual fields.

What are the underlying cognitive mechanisms that modulate visual field biases? The parietal cortex is involved in many different cognitive processes. Two of these processes, remembering stimuli presented at each trial and controlling task settings across a block of trials, are often confounded in VSTM experiments that present stimuli in a blocked fashion. In order to disentangle the effects of these two task demands on dynamic reallocation of resources, it is necessary to demonstrate a change in bias that occurs within conditions that normally do not result in visual field asymmetries. One of the ways to do so is to manipulate context. The logic is as follows: if re-allocation of resources to the right visual field occurs in preparation for increased task demands from a lessdemanding (single-feature VSTM) to a more-demanding (two-feature VSTM) condition, then a right visual-field benefit should be seen when trials of the less-demanding condition are embedded within a block of more-demanding condition. Furthermore, the context effect should occur even when visual field biases cannot be measured in either the first or second condition.

We therefore set out to directly manipulate task-set to test the predictions of the dynamic-reallocation theory. In the attention literature, studies have successfully manipulated behavioral performance by inducing top-down task sets (Folk, Leber, \& Egeth, 2008). To determine whether visual field asymmetries reflect the dynamic reallocation of resources across the visual field, we borrowed from this logic, using task set to manipulate the observer's expectation, and therefore modulate the allocation of right hemisphere resources in VSTM. We reasoned that if visual field asymmetries reflect the dynamic reallocation of resources by IPS, then performance in each visual field should reflect not only current task demands, but also preparation for the upcoming task. We manipulated task set by measuring performance for singlefeature memory trials embedded within a condition that induces resource reallocation to the right visual field. The dynamic reallocation resource theory predicts that inducing reallocation of right hemisphere resources to the right visual field should result in a benefit in VSTM performance in the right visual field as compared to single-feature trials embedded in single-feature blocks. In other words, because trial demands remain the same, any difference in performance reflects reallocation of resources by task-set to the right visual field, inducing an improvement in performance in the right visual field.

In a set of two experiments, we asked whether visual field asymmetries are driven by participants' task set, rather than stimulus related processing. In particular, task set should modulate VSTM performance for single-feature trials that, under normal circumstances, do not demonstrate measurable asymmetries across the visual field (Sheremata \& Shomstein, 2014). To modulate VSTM task set, performance was measured for single-feature trials under two difference context scenarios: (i) embedded among other single-feature trials; and (ii) embedded among mostly two-feature trials. 
The prediction is as follows: if the right hemisphere allocates resources to the right visual field across two-feature blocks to prepare for increased task demands, then there should be improved performance for single-feature trials embedded in twofeature blocks in the right, but not left, visual field. In contrast, if visual field asymmetries simply reflect differences in stimulus-driven resources, then performance on singlefeature trials should be similar regardless of task set (i.e., left visual field benefit no matter the context).

To foreshadow our results, two-feature task set induced improved performance in the right visual field for performance in single-feature memory trials. By inducing right visual field benefits in single-feature trials, we demonstrate that resource allocation across the visual field is under top-down control. These results demonstrate that VSTM asymmetries across the visual field engage flexible allocation of resources for upcoming task demands, linking behavioral performance in VSTM to neural mechanisms occurring in IPS.

\section{Methods}

\section{Participants}

A total of 25 (Experiment 1) and 38 (Experiment 2) righthanded participants, naïve to the purpose of the study, completed experiments for course credit. Sample size was determined using power analyses from a previous report (Sheremata \& Shomstein, 2014) that employed similar stimuli and task demands. Participants ranged in age from 18 years to 23 years, had normal or corrected-to-normal vision, and gave informed consent approved by the George Washington Institutional Review Board.

\section{Apparatus and task}

Experiments were conducted using the Psychopy software package (Peirce, 2009). Stimuli were presented on a 21-inch color monitor $(1,280 \times 1,024$ pixels, $75 \mathrm{~Hz}$ refresh rate $)$, at a viewing distance of approximately $62 \mathrm{~cm}$.

Participants performed a change-detection task in which three colored shapes were presented against a mean gray luminance background. Maximally discriminable, common colors (dark blue, orange red, green, yellow, purple, plum, and maroon) and shapes (rhombus, cross, triangle, diamond, circle, club, and star) were pseudorandomly chosen without replacement. In Experiment 1, during single-feature trials, all items were presented in a single value of the irrelevant feature dimension, pseudorandomly chosen. For example, on shape trials, shapes varied but all the shapes were drawn in a single color and would not change over the memory delay (e.g., dark blue triangle, circle, and star). On the other hand, on color trials, the same shape was presented (e.g., dark blue, green, and plum crosses) (Fig. 1a). The only aspect different in Experiment 1 and Experiment 2, is that, in Experiment 2, each stimulus always had a unique value for both the relevant and irrelevant feature, and a pre-stimulus cue differentiated singleand two-feature trials (Fig. 3a). In both experiments, color and shape both varied on two feature trials.

Stimuli subtended $1.2^{\circ}$ of visual angle along the longest dimension and were presented on a perimeter of an imaginary circle with a radius of $5^{\circ}$ from fixation, rendering all items equidistant from the fixation point. In half of the blocks, items were presented left of fixation, and, in the other half of the blocks, items were presented right of fixation, with visual field order counterbalanced across participants. Participants were instructed to maintain fixation, and, before each trial, the fixation cross blinked off and then on to redirect gaze toward fixation.

On each trial, a memory array was presented for $500 \mathrm{~ms}$, followed by a 1,000 ms memory delay (Fig. 1a). After the delay, the items were again presented. In half of the trials, one of the items changed in the relevant dimension (one-feature: color, shape; two-feature: either color or shape), and on the other half there was no change. A verbal cue $(500 \mathrm{~ms}$, color, shape, or either) directed participants to remember color, shape or either color or shape (color, shape, or either) followed by a $200 \mathrm{~ms}$ blank screen, and participants were asked to indicate whether all items remained the same or if there was a change in one of the items' relevant dimension. Auditory feedback was given after each trial to indicate the accuracy of the response.

The number of to-be-remembered features was manipulated in blocks of 20 trials, with eight two-feature and four single-feature blocks in each visual hemifield. In singlefeature blocks, participants were cued to remember the color or the shape of items within the memory array in all trials. In two-feature blocks, participants were instructed that either color or shape of one of the objects would change in $80 \%$ of the trials. In the remaining $20 \%$ of the trials, referred to as the critical trials, the cue indicated which feature dimension (color or shape) would vary. Each two-feature block of 20 trials was comprised of four mini-blocks of five trials in which the critical trial was the third or the fourth trial (Fig. 1b). In the twofeature blocks, performance was measured only for the critical trials as the demands of each trial reflected the single-feature condition, but task-set reflected the two-feature condition.

\section{Analysis}

Performance for single-feature trials was measured in terms of sensitivity (d'). For trials within two-feature blocks, sensitivity was measured from the 32 single-feature critical trials embedded within the two-feature blocks. For the single-feature blocks, d' was measured for all of the trials for the singlefeature blocks. For each participant, capacity was also 
A

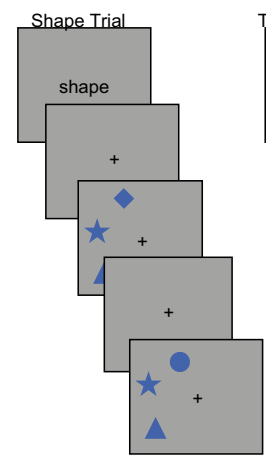

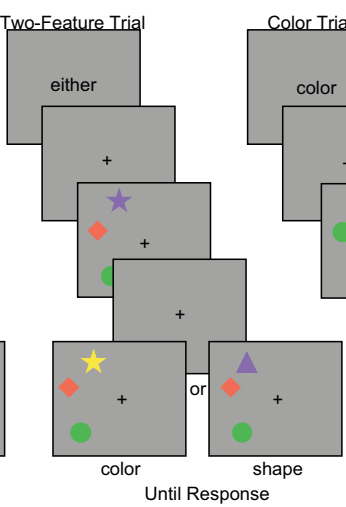

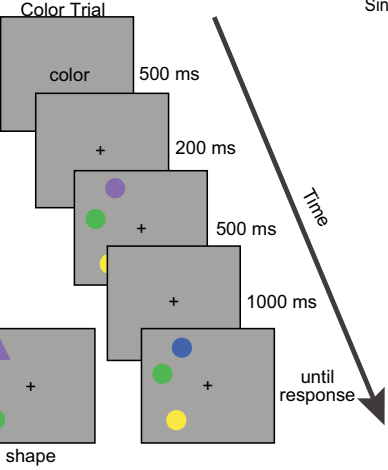

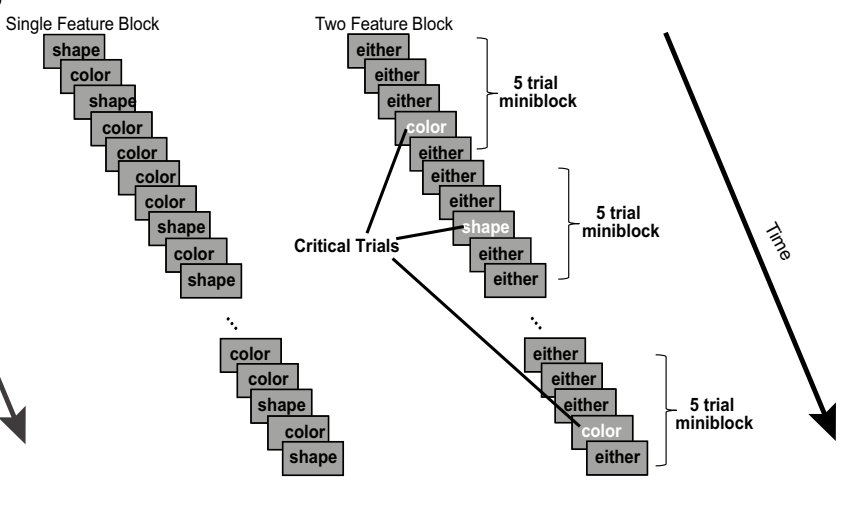

Fig. 1 a Stimuli and visual short-term memory (VSTM) trial structure for single-feature (color or shape) and two-feature (either color or shape) trials. b Mini-block structure for single- and two-feature blocks. In singlefeature blocks, a cue indicated whether the color or shape was the relevant feature dimension (100\% of the trials). In two-feature blocks,

measured using Pashler's formula, $\mathrm{K}=\mathrm{SS} *((\mathrm{H}-\mathrm{FA}) /(1-$ FA)), where SS represents set size, $\mathrm{H}$ represents hit rate, and FA represents false alarm rate (Pashler, 1988) for the singlefeature trials, and any participant with a $\mathrm{K}$ value of less than two objects in either hemifield was excluded due to poor performance.

\section{Results}

In Experiment 1, we sought to measure the effect of task set on the reallocation of resources across the visual field. Participants unable to remember at least two items (two participants), and those who failed to follow experimental protocol (talking on a cell phone during the experiment, one participant) were excluded from the data analysis. Data from the remaining 22 participants were analyzed using an omnibus ANOVA with factors of visual field and task set. Resource reallocation was estimated by measuring the change in sensitivity (d') for critical single-feature VSTM trials embedded in two-feature blocks as compared to single-feature blocks.

If task set induces a dynamic reallocation of resources in VSTM, the two-feature task set should result in a shift of resources toward the right visual field resulting in performance benefits for critical trials embedded within twofeature blocks in the right visual field as compared to trials within the single-feature blocks. Consistently, an omnibus ANOVA with factors of visual field and task set revealed a significant interaction between visual field and task set $[\mathrm{F}(1$, 21) $=4.70, P=0.04, \eta_{p}{ }^{2}=0.20$; Fig. 2]. Importantly, the interaction reflected that the two-feature task set induced a significant improvement for single-feature memory performance when items were presented in the right [single feature task set, d' $=2.32$, two-feature task set, d' $=2.83 ; \mathrm{t}(21)=2.81$, performance was measured for trials in which color or shape was cued (20\%), embedded amongst trials in which participants were cued to detect a change in either color or shape (80\%). In the two-feature block, singlefeature trials followed two two- or three two-feature trials to induce a twofeature task set

$P=0.01$ ) but not left (single feature task set, d' $=2.50$; twofeature task set, $\left.\mathrm{d}^{\prime}=2.44 ; \mathrm{t}(21)=0.31, P=0.76\right)$ visual field. These results strongly suggest that memory task demands led to allocation of additional resources for memory items in the right visual field, consistent with feature-load dependent dynamic reallocation of memory to the right visual field.

A previous report (Sheremata \& Shomstein, 2014) showed that performance on the two-feature task was significantly poorer in two-feature trials as compared to singlefeature trials in the left, but not right, visual field, demonstrating a visual hemifield asymmetry in feature cost. While it is possible that performance on two-feature trials here is contaminated by inclusion of critical trials during the two-feature blocks, we went ahead and analyzed the data to determine whether there was evidence for a redistribution of resources in the two-feature trials as well as in the critical trials.

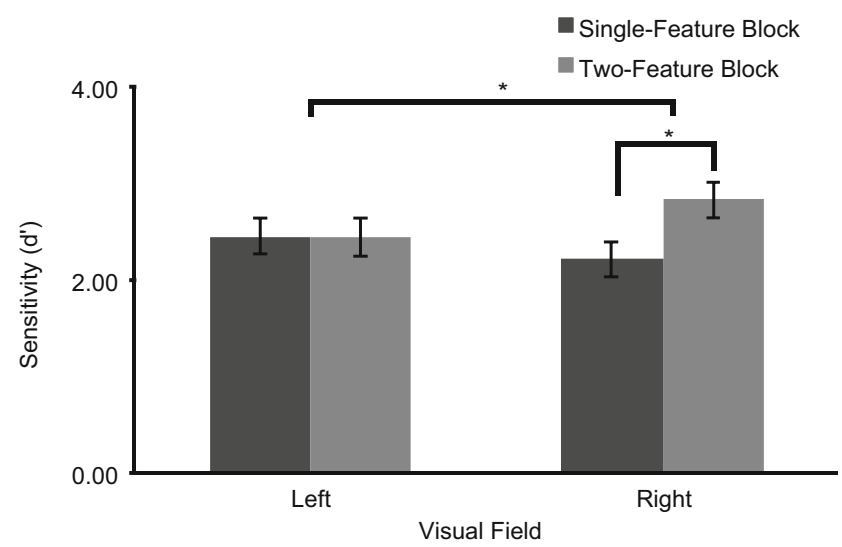

Fig. 2 Results from Experiment 1 demonstrated that performance for single-feature trials demonstrated a task-set performance benefit that depended upon visual field. In the right visual field, sensitivity for detecting a change was enhanced for single-feature trials embedded in twofeature as compared to single-feature blocks, demonstrating an effect of two-feature task set on performance in the right, but not left, visual field 
Consistent with the prediction and replicating our results from Sheremata \& Shomstein (2014), in Experiment 1 there was a significant feature cost in the left $\left[\mathrm{t}_{(22)}=3.27, P<0.01\right)$ but not in the right $\left[\mathrm{t}_{(22)}=1.85, P>0.05\right]$ visual hemifield.

It could be argued, however, that because the stimuli were perceptually different between the single-feature and twofeature conditions, visual-field asymmetries reflect perceptual or attention processes unrelated to memory. A second set of participants therefore performed a change detection task in which stimuli were held constant across the single-feature and two-feature blocks.

In Experiment 2, the task was identical to that in Experiment 1, except that, in single-feature trials, both the relevant and irrelevant feature dimension varied in each trial (Fig. 3a). Six participants' data were excluded because of inability to remember at least two objects (five participants) or failure to follow experimental protocol (one participant). The remaining data were analyzed using an omnibus ANOVA with factors of visual field and task set. A significant main effect of task set $\left[\mathrm{F}(1,31)=4.24, P<0.05, \eta_{p}{ }^{2}=0.22\right]$ reflected better overall performance for single-feature trials embedded in the two-feature blocks $[\mathrm{t}(31)=2.05, P<0.05]$ (Fig. 3b).

Importantly, and consistent with the results from Experiment 1, there was a significant interaction between visual field and task set $[\mathrm{F}(1,31)=4.64, P=$ $\left.0.04, \eta_{p}{ }^{2}=0.10\right)$, reflecting a significant task-set benefit for remembered items in the right- but not left- visual field. Consistent with the reallocation of resources by memory task set, these results demonstrate that twofeature task set resulted in a memory performance benefit in the right visual field [single feature task set, d' = 2.18 , two-feature task set, $\mathrm{d}^{\prime}=2.58 ; \mathrm{t}(31)=2.73, P<$ $0.05]$, but not in the left visual field (single feature task set, $\mathrm{d}^{\prime}=2.23$, two-feature task set, $\mathrm{d}^{\prime}=2.30 ; \mathrm{t}(31)=$ $0.54, P=0.55)$ that cannot be accounted for by perceptual task demands.

To determine whether task-set also demonstrated an asymmetry in the two-feature trials, feature cost was measured by comparing single-feature and two-feature trials. In contrast to Experiment 1, however, there was a significant feature cost in both the left- $\left[\mathrm{t}_{(32)}=4.08, P\right.$ $<0.001]$ and right- $\left[\mathrm{t}_{(32)}=3.69, \mathrm{p}<0.001\right]$ visual hemifield. The difference between experiments is likely due to the difference in strength of reallocation of resources between the two experiments, or increased variability across trials due to missed cue information in the two-feature condition in Experiment 2. Furthermore, Sheremata \& Shomstein (2014) did not find an asymmetry in two-feature trials due to differences in cognitive demands across participants when set size was the same across participants with different memory capacities.

\section{Discussion}

Here, we establish that task resources are differentially and dynamically allocated across the visual field in preparation for remembering single- and multiple-feature objects. Specifically, we induced right visual field benefits by modulating participants' task set, allowing VSTM to optimize the allocation of resources according to the task at hand. We reasoned that, if allocation of resources is fixed, then performance should be constant regardless of task set. If, on the other hand, allocation of resources is flexible, then resources can be distributed across the visual field in a task-specific manner. By manipulating task set while measuring performance for the same single-feature condition (i.e., critical trial
A

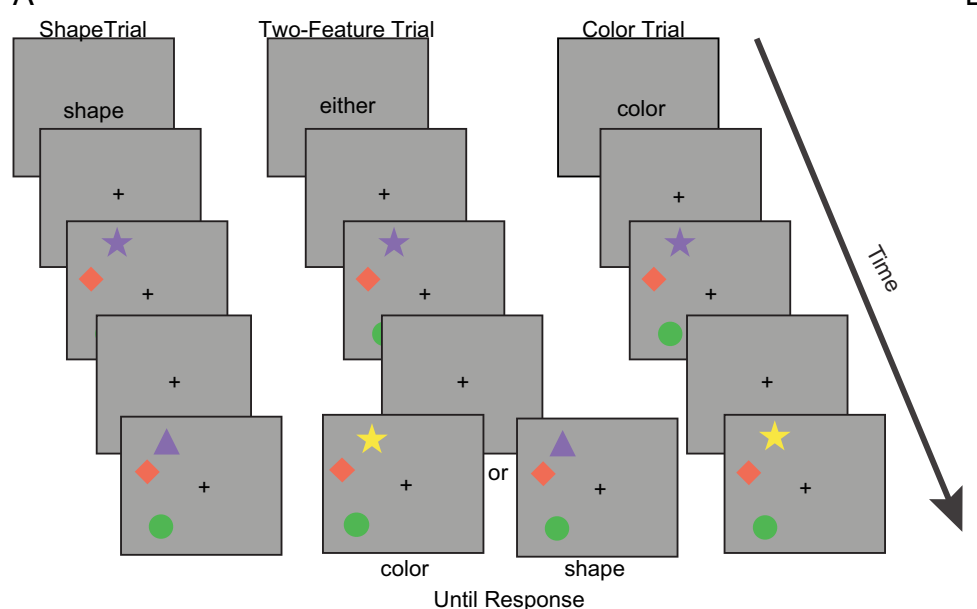

B

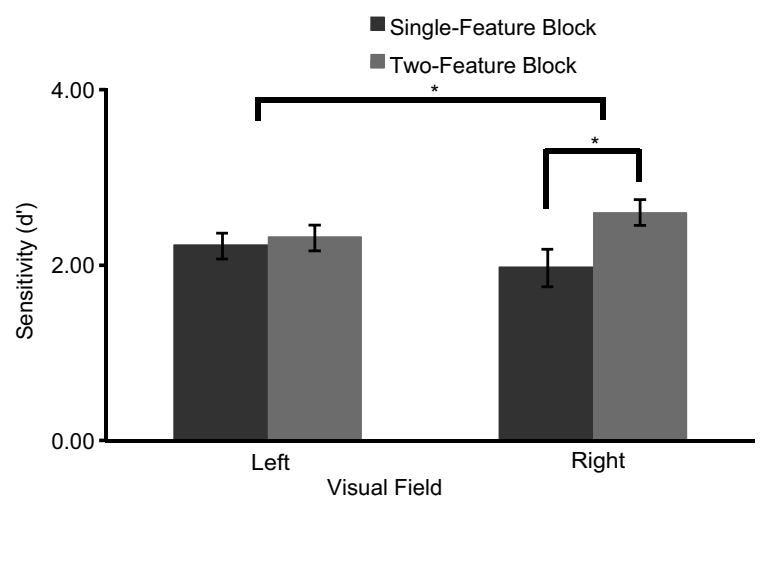

Fig. 3 a In Experiment 2, stimuli were identical across trial types, and pre-stimulus cues differentiated single-feature and two-feature trials. b Mirroring the results from Experiment 1, single-feature trials in the right visual field demonstrated a benefit when embedded in two-feature trials, demonstrating that perceptual demands could not account for performance differences in Experiment 1 
was the same and what varied was the task context), we were able to induce changes in how VSTM was represented across the visual field. As predicted by the dynamic reallocation of resources hypothesis (Sheremata \& Shomstein, 2014), performance on single-feature trials embedded in a two-feature task set led to a systematic shift of resources to the right visual field, resulting in an improvement in VSTM performance in the right, but not left, visual field. These results demonstrate that manipulating participants' task set is sufficient to induce right visual field biases, elucidating a mechanism by which resources can be redistributed with increasing memory load.

It is important to note that, in the current studies, visual field asymmetries were not expected at a single set size of three memory items. Sheremata \& Shomstein (2014) found significant visual field asymmetries when set sizes were determined based upon individual participants' capacity (i.e. $\mathrm{K}_{\mathrm{p}}+1$ ), likely due to equating task difficulty across individuals. Therefore, we did not expect to see visual field asymmetries using a single set size. In contrast, embedding single-feature trials in two-feature blocks led to an improvement in the right visual field even within a condition that typically does not result in visual field asymmetries, consistent with the dynamic reallocation theory.

One intriguing prediction is that if dynamic reallocation of resources results in a redistribution from the leftto the right-visual field, performance should decrease for trials presented in the left-visual field. While this may be expected when there is competition between the two visual fields, visual field was blocked in the current experiments. Left-visual field performance was never tested during a condition in which resources were reallocated to the right visual field. From the current findings, however, it would be predicted that manipulating task-set should result in a decrement in performance for leftvisual field trials embedded in right- versus left- visual field blocks.

These studies demonstrate, for the first time, that topdown influences modulate resource distribution across the visual field. By constraining memory demands, any performance differences across the visual field can only be attributed to task set and are not based on trial-by-trial task demands, demonstrating that preparation for remembering two-features is sufficient to induce the reallocation of resources across the visual field. Task set induced right visual field benefits for a discrete set size (three objects) even though previously no difference across the visual field had been reported at this memory load. These results suggest that differences in resource allocation are present even when they cannot be measured within a single condition. Comparing performance across conditions demonstrated that top-down factors modulated the deployment of resources across the visual field.
Within the fronto-parietal cortex, areas of the posterior parietal cortex demonstrate activity involved in preparation for current task demands (Corbetta \& Shulman, 2002; Kastner \& Ungerleider, 2000; Sohn, Ursu, Anderson, Stenger, \& Carter, 2000). These areas also demonstrate task-dependent asymmetries during visual attention (Sheremata \& Silver, 2015; Szczepanski et al., 2010) and short-term memory (Sheremata et al., 2010). The current results show that task set, an important component of task preparation that relies on IPS, and therefore overlaps with VSTM in the brain, modulates behavior in a visual-field dependent manner. Specifically, reallocation of resources reflects the neural processes underlying task preparation.

Early studies specifically investigated the role of the parietal cortex in VSTM, demonstrating that posterior parietal cortex mirrored memory capacity (Todd \& Marois, 2004), and reflected the complexity of items held in memory (Xu \& Chun, 2006). More recent reports have shown that the pattern of BOLD signal in early visual cortex can discriminate between a set of stimuli held in memory (Emrich, Riggall, LaRocque, \& Postle, 2013; Harrison \& Tong, 2009; Serences, Ester, Vogel, \& Awh, 2009), suggesting that the representation may be stored in early visual cortex. However, there is some disagreement as to whether the same information is maintained in parietal cortex (Bettencourt \& Xu, 2016; Ester, Sprague, \& Serences, 2015), or whether the representation is stored solely in early visual cortex (Riggall \& Postle, 2012). While the exact role of individual cortical areas in VSTM may not be entirely clear, disruption to right parietal cortex impairs change detection performance across the visual field (Beck, Muggleton, Walsh, \& Lavie, 2006), demonstrating that parietal cortex plays an integral role in the cognitive mechanisms underlying VSTM.

Similarly, one may question whether dynamic reallocation reflects the transfer of discrete representations (Zhang \& Luck, 2008) or flexible resources (Bays \& Husain, 2008) across the visual field. As designed, the current studies cannot distinguish between the slot and resource models of VSTM. Future investigations of visual field asymmetries, however, may help to distinguish these two theories of short-term memory.

Our results set up clear hypotheses to test how each hemisphere contributes to the maintenance of VSTM representations. Areas of the parietal cortex, shown to be involved in VSTM (Todd \& Marois, 2004; Xu \& Chun, 2006), do not simply direct resources to the contralateral visual field. Instead the right hemisphere can direct its resources across the visual field in a loaddependent manner (Sheremata et al., 2010). Areas of the parietal cortex that demonstrate memory-load 
dependent hemispheric asymmetries show feature-load dependence (Xu \& Chun, 2006). It is likely, therefore, that visual field asymmetries reflect asymmetric processing between left and right parietal cortex. Our findings further the understanding of how these asymmetries emerge, and point to the role of top-down modulation of stored representations in VSTM.

Acknowledgments This work was supported by grants from the National Science Foundation (BCS-1059523) and National Institutes of Health (R21-EY021644) to S. Shomstein.

\section{References}

Alvarez, G. A., \& Cavanagh, P. (2004). The capacity of visual short-term memory is set both by visual information load and by number of objects. Psychological Science, 15(2), 106-111. doi:10.1111/j.09637214.2004.01502006.x

Bays, P. M., \& Husain, M. (2008). Dynamic shifts of limited working memory resources in human vision. Science, 321(5890), 851-854. doi:10.1126/science. 1158023

Beck, D. M., Muggleton, N., Walsh, V., \& Lavie, N. (2006). Right parietal cortex plays a critical role in change blindness. Cerebral Cortex, 16(5), 712-717. doi:10.1093/cercor/bhj017

Behrmann, M., Watt, S., Black, S. E., \& Barton, J. (1997). Impaired visual search in patients with unilateral neglect: An oculographic analysis. Neuropsychologia, 35(11), 1445-1458. doi:10.1016/S0028-3932(97)00058-4

Bettencourt, K. C., \& Xu, Y. (2016). Decoding the content of visual shortterm memory under distraction in occipital and parietal areas. Nature Neuroscience, 19(1), 150-157. doi:10.1038/nn.4174

Bisiach, E., \& Vallar, G. (1988). Hemineglect in humans. In Handbook of neuropsychology, Vol. 1 (pp. 195-222). Elsevier Science.

Bowers, D., \& Heilman, K. M. (1980). Pseudoneglect: Effects of hemispace on a tactile line bisection task. Neuropsychologia, 18, 491-498.

Corbetta, M., \& Shulman, G. L. (2002). Control of goal-directed and stimulus-driven attention in the brain. Nature Reviews Neuroscience, 3(3), 201-215. doi:10.1038/nrn755

Cowan, N. (2010). The magical mystery four how is working memory capacity limited, and why? Current Directions in Psychological Science, 19(1), 51-57. doi:10.1177/0963721409359277

Eglin, M., Robertson, L. C., \& Knight, R. T. (1989). Visual search performance in the neglect syndrome. Journal of Cognitive Neuroscience.

Emrich, S. M., Riggall, A. C., LaRocque, J. J., \& Postle, B. R. (2013). Distributed patterns of activity in sensory cortex reflect the precision of multiple items maintained in visual short-term memory. Journal of Neuroscience, 33(15), 6516-6523. doi:10.1523/JNEUROSCI.573212.2013

Ester, E. F., Sprague, T. C., \& Serences, J. T. (2015). Parietal and frontal cortex encode stimulus-specific mnemonic representations during visual working memory. Neuron, 87(4), 893-905. doi:10.1016/j. neuron.2015.07.013

Folk, C. L., Leber, A. B., \& Egeth, H. E. (2008). Top-down control settings and the attentional blink: Evidence for nonspatial contingent capture. Visual Cognition.

Fougnie, D., Asplund, C. L., \& Marois, R. (2010). What are the units of storage in visual working memory? Journal of Vision, 10(12), 27. doi: $10.1167 / 10.12 .27$
Gamble, C. M., \& Somers, D. C. (2012). An Emergent Hemifield Asymmetry for Visual Short-Term Memory Capacity. Journal of Vision, 12, 347-347.

Harrison, S. A., \& Tong, F. (2009). Decoding reveals the contents of visual working memory in early visual areas. Nature, 458(7238), 632-635. doi:10.1038/nature07832

Kastner, S., \& Ungerleider, L. G. (2000). Mechanisms of visual attention in the human cortex. Annual Review of Neuroscience, 23, 315-341. doi:10.1146/annurev.neuro.23.1.315

Kinsbourne, M. (1987). Mechanisms of unilateral neglect. In Neurophysiological and neuropsychological aspects of spatial neglect (Vol. 45, pp. 69-86). Elsevier. doi:10.1016/S0166-4115(08)61709-4

Luck, S. J., \& Vogel, E. K. (1997). The capacity of visual working memory for features and conjunctions. Nature, 390(6657), 279-281. doi: $10.1038 / 36846$

Mesulam, M.-M. (1999). Spatial attention and neglect: Parietal, frontal and cingulate contributions to the mental representation and attentional targeting of salient extrapersonal events. Philosophical Transactions of the Royal Society, B: Biological Sciences, 354(1387), 1325-1346. doi:10.1098/rstb.1999.0482

Pashler, H. (1988). Familiarity and visual change detection. Perception \& Psychophysics, 44(4), 369-378. doi:10.3758/BF03210419

Peirce, J. W. (2009). Generating stimuli for neuroscience using PsychoPy. Frontiers in Neuroinformatics, 2.doi:10.3389 /neuro.11.010.200810.3758/s13423-016-1181-8

Phillips, W. A. (1974). On the distinction between sensory storage and shortterm visual memory, 16(2), 283-290. doi: 10.3758/BF03203943

Posner, M. I., Walker, J. A., Friedrich, F. J., \& Rafal, R. D. (1984). Effects of parietal injury on covert orienting of attention. Journal of Neuroscience, 4(7), 1863-1874.

Poynter, W., \& Roberts, C. (2012). Hemispheric asymmetries in visual search. Laterality: Asymmetries of Body, Brain and Cognition, 17(6), 711-726. doi:10.1080/1357650X.2011.626558

Riggall, A. C., \& Postle, B. R. (2012). The relationship between working memory storage and elevated activity as measured with functional magnetic resonance imaging. The Journal of Neuroscience, 32(38), 12990-12998. doi:10.1523/JNEUROSCI.1892-12.2012

Serences, J. T., Ester, E. F., Vogel, E. K., \& Awh, E. (2009). Stimulus-specific delay activity in human primary visual cortex. Psychological Science, 20(2), 207-214. doi:10.1111/j.1467-9280.2009.02276.x

Sheremata, S. L., Bettencourt, K. C., \& Somers, D. C. (2010). Hemispheric asymmetry in visuotopic posterior parietal cortex emerges with visual short-term memory load. The Journal of Neuroscience. doi:10.1523/JNEUROSCI.2689-10.2010

Sheremata, S., \& Shomstein, S. (2014). Hemifield asymmetries differentiate VSTM for single-and multiple-feature objects. Attention, Perception \& Psychophysics. doi:10.3758/s13414-014-0689-0

Sheremata, S. L., \& Silver, M. A. (2015). Hemisphere-dependent attentional modulation of human parietal visual field representations. The Journal of Neuroscience. doi:10.1523/JNEUROSCI.2378-14.2015

Sohn, M.-H., Ursu, S., Anderson, J. R., Stenger, V. A., \& Carter, C. S. (2000). The role of prefrontal cortex and posterior parietal cortex in task switching. Proceedings of the National Academy of Sciences, 97(24), 13448-13453. doi:10.1073/pnas.240460497

Szczepanski, S. M., Konen, C. S., \& Kastner, S. (2010). Mechanisms of spatial attention control in frontal and parietal cortex. Journal of Neuroscience, 30(1), 148-160. doi:10.1523/JNEUROSCI.386209.2010

Thiebaut de Schotten, M., Dell'Acqua, F., Forkel, S. J., Simmons, A., Vergani, F., Murphy, D. G. M., \& Catani, M. (2011). A lateralized brain network for visuospatial attention. Nature Neuroscience, 14, 1245-1246.

Todd, J. J., \& Marois, R. (2004). Capacity limit of visual short-term memory in human posterior parietal cortex. Nature. doi:10.1038/nature02466 
Vogel, E. K., \& Machizawa, M. G. (2004). Neural activity predicts individual differences in visual working memory capacity. Nature, 428 , 748-751. doi:10.1038/nature02447

Wilson, K. E., Adamo, M., Barense, M. D., \& Ferber, S. (2012). To bind or not to bind: Addressing the question of object representation in visual short-term memory. Journal of Vision, 12(14), 1-16. doi:10.1167/12.8.14
Xu, Y., \& Chun, M. M. (2006). Dissociable neural mechanisms supporting visual short-term memory for objects. Nature, 440(7080), 91-95. doi:10.1038/nature04262

Zhang, W., \& Luck, S. J. (2008). Discrete fixed-resolution representations in visual working memory. Nature, 453(7192), 233-235. doi:10.1038/nature06860 